\title{
Identification of the potential molecular mechanism and driving mutations in the pathogenesis of familial intestinal gastric cancer by whole exome sequencing
}

\author{
HAIXIANG CHEN $^{1 *}$, JUAN WANG $^{2 *}$, YI ZHUANG $^{3}$ and $\mathrm{HAO} \mathrm{WU}^{4}$ \\ ${ }^{1}$ Department of Medical Records, The Third Affiliated Hospital of Nanjing University of Chinese Medicine, Nanjing, \\ Jiangsu 210001; ${ }^{2}$ Department of General Surgery, Xi Jing Hospital, Fourth Military Medical University, Xi'an, \\ Shaanxi 710032; ${ }^{3}$ Department of Respiratory Medicine, The Affiliated Drum Tower Hospital of \\ Nanjing University Medical School, Nanjing, Jiangsu 210008; ${ }^{4}$ Department of Oncology, \\ The First Affiliated Hospital of Nanjing Medical University, Nanjing, Jiangsu 210029, P.R. China
}

Received September 29, 2017; Accepted July 27, 2018

DOI: $10.3892 /$ or.2018.6613

\begin{abstract}
The genetic alterations in familial intestinal gastric cancer (FIGC) have not been clearly understood. Aiming to explore the molecular basis and the driving mutations underlying the pathogenesis of FIGC, we performed exome sequencing of the blood samples of the members of an extended family with FIGC. The differences in mutation patterns between family members with gastric cancer and controls were analysed and the overlapped variants were screened by comparing previously published data for blood and tumours from gastric cancer patients. The overlapped genes harbouring insertions-deletions (INDELs) and single-nucleotide variants (SNVs) were subjected to function, pathway and network analysis. The INDELs were enriched in DNA packaging and in the neurological system process related to the biological process (BP), while SNVs were closely related to cell-function-related BPs. ESR was the significant node with marked centrality in the SNV network. ERK 1/2 was the hub node in the INDEL network, interacting with EZK and IGF2R. Sequencing analysis revealed ESR1 homozygous mutations in exon $1(216 \mathrm{G}>\mathrm{C})$ and exon $10(2234 \mathrm{C}>\mathrm{T})$ and EZR1 heterozygous deletion of 68-69 GT nucleotides in exon 13 of the family members. The IGF2R gene only demonstrated a mutation in exon 48 of the propositus. All hub proteins had direct or indirect interactions in the protein-protein interaction network.
\end{abstract}

Correspondence to: Dr Haixiang Chen, Department of Medical Records, The Third Affiliated Hospital of Nanjing University of Chinese Medicine, 1 Jinling Road, Nanjing, Jiangsu 210001, P.R. China

E-mail: 13905170601@163.com

${ }^{*}$ Contributed equally

Key words: familial intestinal gastric cancer, whole exome sequencing, ingenuity pathway analysis, protein structure modelling

\section{Introduction}

Gastric cancer (GC) is the second most frequent cause of cancer-related deaths worldwide (1). Several studies revealed that a positive family history of having a first-degree relative with $\mathrm{GC}$ is considered a strong risk factor for the development of GC, particularly when two or more relatives are affected (2). There is familial aggregation in $\sim 10-20 \%$ of GCs and $\sim 1-3 \%$ have a clear inherited genetic conditioning (3). A good understanding of the genetic mechanism of GC in the family may shed light on the driving genes and pathways for treatment options and genetic counselling. However, the genetic events that predispose individuals to GC have not been clearly understood.

Three hereditary GC syndromes have been described which are the following: Hereditary diffuse gastric cancer (HDGC), familial intestinal gastric cancer (FIGC) and the recently proposed gastric adenocarcinoma and proximal polyposis of the stomach (GAPPS) (4). Some other hereditary cancer syndromes such as hereditary non-polyposis colorectal cancer (HNPCC), Li-Fraumeni syndrome (LFS), familial adenomatous polyposis (FAP) and Peutz-Jeghers syndrome (PJS) also predispose individuals to GC (5). Genetic and epigenetic alterations play key roles in the pathogenesis of familial GC development (6). Except for HDGC, the molecular basis for the familial aggregation remains largely unknown. Identification of new predisposition genes would provide novel insights regarding the molecular pathogenesis of GC. However, the pathogenesis and genetic changes of FIGC have not been clearly elucidated.

Whole-exome sequencing has been widely used to identify the genomic mutation signatures for uncovering the predisposing genes in familial cancers $(7,8)$. In the present study, we explored three genomic variations (ESR1, IGF2R and EZR) in FIGC by whole-exome sequencing. The oestrogen receptor $\alpha /$ oestrogen receptor 1 (ER $\alpha / E S R 1)$ gene, a well-known proto-oncogene, is a member of the nuclear hormone receptor family and plays an important role in hormone binding, DNA binding and activation of transcription (9). The 
mannose-6-phosphate/insulin-like growth factor 2 receptor (M6P/IGF2R), referred to as IGF2R, is a multifunctional protein ubiquitously expressed in human tissues and has been recently identified as a tumour suppressor (10). Ezrin, encoded by the EZR gene, is a signal transduction component belonging to the ezrin-radixin-moesin (ERM) protein family; it acts both as a link between the actin cytoskeleton and plasma membrane proteins and as a substrate for tyrosine kinase (11).

The detection of these mutations, which appear to predispose individuals to familial GC, could lead to the identification of individuals with a risk of familial GC in affected families and may be useful as biomarkers for confirmatory diagnosis of FIGC and appropriate treatment, providing new insights into tumour initiation and the progression of FIGC. Screening for these genotypes combined with information on the familial background may help us to identify individuals who are at increased risk of FIGC.

\section{Materials and methods}

Patients. The present study was approved by the Ethics Committee of the Third Affiliated Hospital of Nanjing University of Chinese Medicine. All study procedures were performed according to the Declaration of Helsinki ethical principles. Informed consent was obtained from all participating patients.

The proband was a 37 year-old man diagnosed with severe atrophic gastritis in 2014 by gastroscope inspection (Fig. 1). The proband's mother suffered from chronic atrophic gastritis and died of FIGC at the age of 63 years in 2014 in our hospital. His maternal grandfather and grandmother died of GC and lung cancer, respectively. The two elder brothers of his mother were dead from lung cancer and oesophagus cancer. The two younger brothers of his mother presented with chronic atrophic gastritis. His father had no family history of digestive tract diseases and was considered the normal control. The relations between individuals are illustrated in the family pedigree (Fig. 1).

Sample collection and whole-exome sequencing. A total of $5 \mathrm{ml}$ of peripheral whole blood was collected from the proband and the family members listed in the family pedigree (Fig. 1). The blood samples were collected and stored at $-20^{\circ} \mathrm{C}$ before use.The genomic DNA was extracted using a DNA extraction kit (Youcheng Biological Pharmaceutical Technology, Co. Ltd., Jiangsu, China), following the manufacturer's instructions. The library preparation, whole-exome capture and sequencing were performed at Shanghai GeneChem, Co., Ltd. (Shanghai, China). Sequencing was analysed based on the Illumina PE150 platform (Shanghai Jeayea Biotech Co., Ltd., Shanghai, China).

The mean coverage of study samples was $\mathrm{x} 100$. The variant calling files were created by BCFtools and SAMtools (http://samtools.sourceforge.net). All variant annotations were performed using Variant Effect Predictor (VEP) based on the Ensemble database (http://asia.ensembl. org/info/docs/tools/vep/script/index.html).

Whole-genome analysis. Genes harbouring exonic and/or splice site variations were filtered and stratified to single-nucleotide variant (SNV) and insertion-deletion (INDEL) genes in each sample. Subsequently, the variant genes specific to patient samples were selected, which had different calls from the normal genotype and less than two reads in the normal control sample.

The genome variation profiles (accession no. GSE30833) of the blood and tumour tissues of 2 GC patients were downloaded from the public Gene Expression Omnibus (GEO) database (https://www.ncbi.nlm.nih.gov/geo/). The SNVs and INDELs specific to the patients from our dataset and those from the previous dataset specified above were combined to identify the overlapped somatic variants.

Function annotation of overlapped variants. The Database for Annotation, Visualization and Integration Discovery (DAVID) software allows the functional annotation of gene sets in terms of biological process (BP), molecular function (MF), cellular component (CC) and pathway. The overrepresented Gene Ontology (GO) terms in BPs and the predominant pathways were visualized by DAVID software (http://david.abcc.ncifcrf. gov/). A P-value $<0.05$ was set as the cut-off value of significance.

Ingenuity pathway analysis (IPA). IPA can be used to assign the functional information and biological relevance of genes in the context of known BPs, pathways and regulatory networks (12). The canonical pathways involved with variant genes were analysed by IPA software (Ingenuity Systems, Redwood City, CA, USA). A score was calculated to identify aberrant biological functions associated with the gene list.

Protein-protein interaction network analysis. Osprey served as the biological network visual tool and provided the direct and indirect protein interaction pairs (13). The protein-protein interaction network was established by the Osprey network system version 1.2.0 (Human GRID; https://osprey.thebiogrid.org/).

PCR amplification and sequencing of ESR1, ERK and IGF2R. The primers of oestrogen receptor 1 (ESR1), MAPK3/1, mitogen-activated protein kinase 3/1 (ERK1/2) and insulin-like growth factor 2 receptor (IGF2R) genes were designed by Primer 5 and synthesized by Shanghai Sangong Pharmaceutical Co., Ltd. (Shanghai, China). PCR amplification was performed with the KAPA Taq Extra system (Shanghai Jeayea Biotech Co., Ltd.) in an ABI9700 PCR machine (Applied Biosystems; Thermo Fisher Scientific, Inc., Waltham, MA, USA). The PCR conditions were $94^{\circ} \mathrm{C}$ for $3 \mathrm{~min}, 35$ cycles at $94^{\circ} \mathrm{C}$ for $20 \mathrm{sec}$, $58^{\circ} \mathrm{C}$ for $15 \mathrm{sec}$ and $72^{\circ} \mathrm{C}$ for $3 \mathrm{sec}$, followed by a final elongation step at $72^{\circ} \mathrm{C}$ for $3 \mathrm{~min}$. After amplification, the PCR products were evaluated and sequenced on an ABI 3730XL automated sequencer (Applied Biosystems; Thermo Fisher Scientific, Inc.).

Three-dimensional protein structure prediction. The Expert Protein Analysis System (ExPASy) is a web server for proteomics and protein analysis (14). Based on sequencing the genes of interest (ESR1, ERK and IGF2R), the nucleotide (DNA) sequences were translated into protein sequences using the Translate tool of ExPASy (http://web.expasy.org/translate/). The target amino acid sequences were submitted to SWISS-MODEL (https://swissmodel.expasy.org/interactive/) to produce the final 3-dimensional (3D) protein structure. 


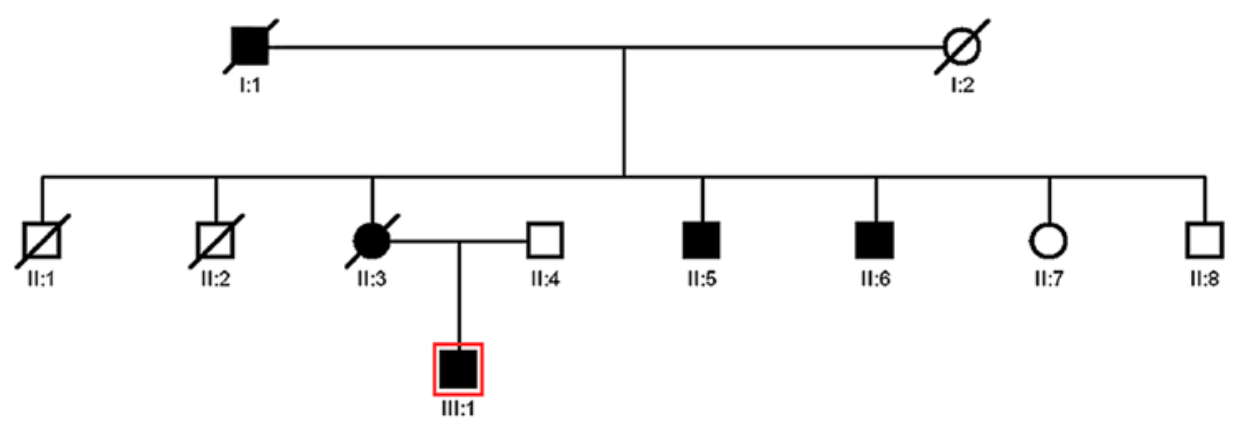

Family pedigree

Figure 1. Family pedigree of the familial intestinal gastric cancer family members. Shaded individuals are diagnosed with gastric cancer, gastric disease or other cancers. Female and male individuals are depicted as circles and rectangles, respectively. Deceased individuals have diagonal lines through their symbol.
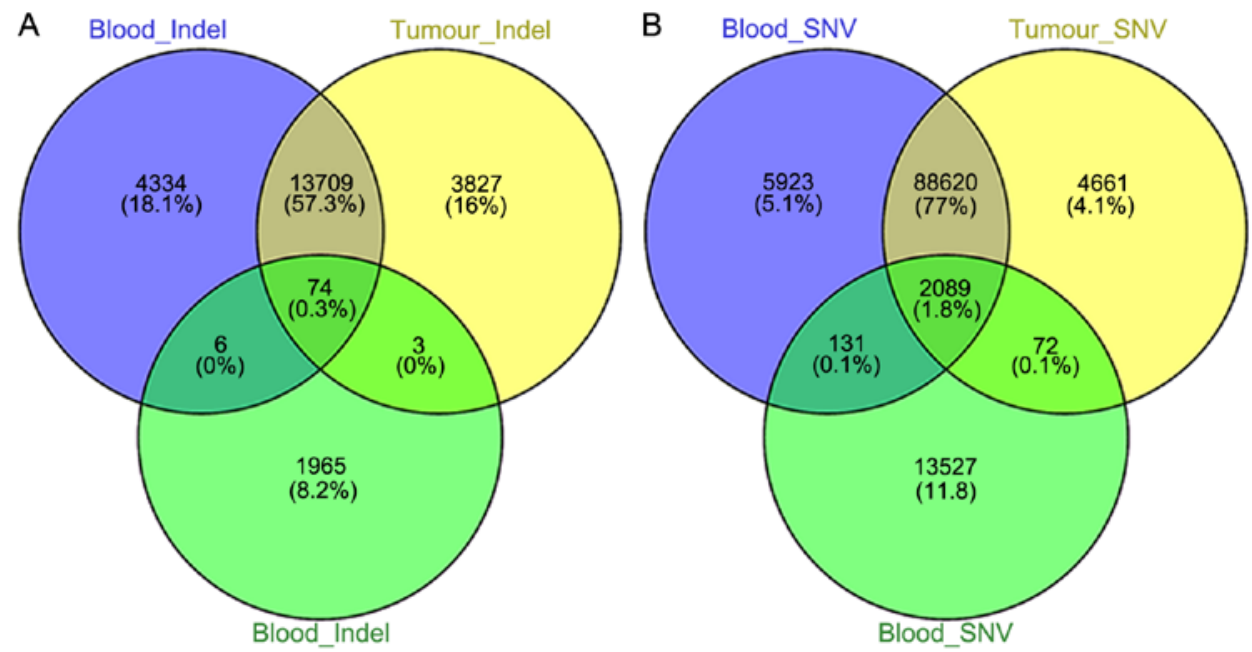

Figure 2. Venn diagram of the overlapped variant genes in the blood samples of family members with familial intestinal gastric cancer. (A) Overlapped INDELs. (B) Overlapped SNVs. Green indicates the variants from blood samples in the present study; blue and yellow indicate the variants from blood and tumour samples from the previous public study. INDELs, insertions-deletions; SNVs, single-nucleotide variants.

Immunofluorescence assay. After being embedded in paraffin, the gastric biopsy specimens of the proband were cut into consecutive $4-\mu \mathrm{m}$ sections. The sections were incubated with the primary anti-IGF2R antibody (1:50; ab32815; Abcam, Cambridge, MA, USA) and anti-ESR1 antibody (1:40; MA5-13304; Thermo Fisher Scientific, Inc., Waltham, MA, USA) overnight at $4{ }^{\circ} \mathrm{C}$. The sections were washed with phosphate-buffered saline (PBS) and incubated with fluorochrome-conjugated secondary antibodies, goat anti-rabbit IgG H\&L (DyLight ${ }^{\circledR}$ 594) (1:200; ab96885; Abcam) and rabbit anti-mouse IgG H\&L (Alexa Fluor ${ }^{\circledR} 488$ ) (1:200; ab150125; Abcam), for $1 \mathrm{~h}$ at $37^{\circ} \mathrm{C}$. The immunofluorescence staining was observed under a fluorescence microscope (Olympus Corp., Tokyo, Japan).

\section{Results}

Data summary of the exome sequencing. In total, $571.94 \mathrm{M}$ of raw reads were generated from the exome sequencing. Following quality control, $565.38 \mathrm{M}$ of effective reads remained. In each sample, there were $>92 \%$ of bases with a Q-value $\geq 30$ and $>96.5 \%$ of bases with a Q-value $\geq 20$. Finally, we obtained 2048 INDELs and 15819 SNVs by exome sequencing.
Overlapped SNVs and INDELs. A Venn diagram is a simple and effective procedure that displays the overlapped gene list from different groups (15). The overlapped variant genes, compared with the public exome sequencing data of the blood and tissue samples of GC patients, are displayed in Fig. 2 . The overlapped INDELs and SNVs were identified to be 74 and 2089, respectively, for further analysis.

Significant GO terms and pathways. To understand the function of gene variants at the molecular level, the SNVs and INDELs were subjected to GO and pathway enrichment analysis, respectively. As displayed in Table I, INDELs were closely associated with DNA packaging, neurological system processes and BPs related to nucleosome assembly. The significant pathways for INDELs included regulation of the actin cytoskeleton, systemic lupus erythematosus and natural-killer-cell-mediated cytotoxicity. The cell-function-related BPs were perturbed by SNVs, such as cell adhesion, motility and motion. The pathways related to cancers such as bladder, non-small cell lung, thyroid and endometrial cancer were significantly enriched by SNVs (Table I).

IPA network analysis. To identify the potential molecular function and pathways perturbed by gene variations, SNVs and 
Table I. Top 10 significant GO and pathway terms associated with INDELs and SNVs.

\begin{tabular}{|c|c|c|c|}
\hline Gene variants & Term & Count & $\mathrm{P}$-value \\
\hline \multirow[t]{10}{*}{ Indels } & GO:0006323 DNA packaging & 4 & 0.008699692 \\
\hline & GO:0050877 neurological system process & 10 & 0.027926677 \\
\hline & GO:0006334 nucleosome assembly & 3 & 0.037114373 \\
\hline & GO:0031497 chromatin assembly & 3 & 0.039558685 \\
\hline & GO:0065004 protein-DNA complex assembly & 3 & 0.042910555 \\
\hline & GO:0050890 cognition & 8 & 0.044035891 \\
\hline & GO:0034728 nucleosome organization & 3 & 0.044625241 \\
\hline & GO:0016567 protein ubiquitination & 3 & 0.06905842 \\
\hline & GO:0007600 sensory perception & 7 & 0.070872214 \\
\hline & GO:0043087 regulation of GTPase activity & 3 & 0.073137761 \\
\hline \multirow[t]{3}{*}{ KEGG } & hsa04810: Regulation of actin cytoskeleton & 4 & 0.038043588 \\
\hline & hsa05322: Systemic lupus erythematosus & 2 & 0.298474207 \\
\hline & hsa04650: Natural killer cell mediated cytotoxicity & 2 & 0.379898987 \\
\hline \multirow[t]{9}{*}{ SNVs } & GO:0007155 cell adhesion & 79 & $3.99 \mathrm{E}-06$ \\
\hline & GO:0022610 biological adhesion & 79 & $4.23 \mathrm{E}-06$ \\
\hline & GO:0048870 cell motility & 42 & $1.47 \mathrm{E}-05$ \\
\hline & GO:0051674 localization of cell & 42 & $1.47 \mathrm{E}-05$ \\
\hline & GO:0000902 cell morphogenesis & 44 & $1.03 \mathrm{E}-04$ \\
\hline & GO:0006928 cell motion & 54 & $1.40 \mathrm{E}-04$ \\
\hline & GO:0032989 cellular component morphogenesis & 47 & $1.61 \mathrm{E}-04$ \\
\hline & GO:0000904 cell morphogenesis involved in differentiation & 32 & $3.86 \mathrm{E}-04$ \\
\hline & GO:0030855 epithelial cell differentiation & 21 & 7.22E-04 \\
\hline \multirow[t]{10}{*}{ KEGG } & hsa04320: Dorso-ventral axis formation & 7 & 0.004403386 \\
\hline & hsa05219: Bladder cancer & 9 & 0.00487317 \\
\hline & hsa04810: Regulation of actin cytoskeleton & 25 & 0.005877286 \\
\hline & hsa05223: Non-small cell lung cancer & 10 & 0.0072363 \\
\hline & hsa04370: VEGF signalling pathway & 12 & 0.008400522 \\
\hline & hsa04360: Axon guidance & 17 & 0.008753884 \\
\hline & hsa05216: Thyroid cancer & 7 & 0.009484706 \\
\hline & hsa05211: Renal cell carcinoma & 11 & 0.014021769 \\
\hline & hsa04960: Aldosterone-regulated sodium reabsorption & 8 & 0.015080678 \\
\hline & hsa05213: Endometrial cancer & 9 & 0.017688902 \\
\hline
\end{tabular}

GO, Gene Ontology; INDELs, insertions-deletions; SNVs, single-nucleotide variants; KEGG, Kyoto Encyclopedia of Genes and Genomes.

INDELs identified in our study, respectively, were subjected to IPA. As displayed in Fig. 3A the ERK1/2 (MAPK1/3) pathway, interacting with EZR and IGF2R (M6P), was the key node in the INDEL network. The major molecules such as ERK1/2, EZR and IGF2R were mainly involved in cell-to-cell signalling and closely related to interaction and connective tissue disorders and developmental disorders. As displayed in Fig. 3B, a network centred on ESR1 was constructed for SNVs. ESR1 was mainly associated with auditory disease, hereditary disorders and neurological disease, with a highest score of 44 .

Protein-protein interaction. The protein interaction network with the major proteins was constructed by Osprey software. In the present study, 4 proteins (ESR1, ERK, EZK and IGF2R) were selected as the origin nodes. CDH1 was also included in particular to analyse the interactions with the four proteins related to GC. As displayed in Fig. 3C, all proteins were assembled in one protein interaction network and had direct or indirect interactions with other proteins. IGF2R demonstrated regulatory interactions with MAPK3 and MAPK1 by interacting with cellular suppressor of E1A-stimulated genes (CREG1), retinoblastoma 1 (RB1) and myelocytomatosis oncogene (MYC). ESR1 directly interacted with MAPK1, and $\mathrm{CDH} 1$ demonstrated a direct interaction with EZR.

Mutations of IGF2R, EZR and ESR1. The mutations of IGF2R, EZR and ESR1 were determined by PCR amplification and sequencing. The ESR1 gene showed homozygous mutations in exon $1(216 \mathrm{G}>\mathrm{C})$ and exon $10(2234 \mathrm{C}>\mathrm{T})$ in the proband patient. An heterozygous deletion of 68-69 GT nucleotides was detected in exon 13 of the EZR1 gene. In addition, there was an heterozygous insertion of the 1100GGGCG GGTACAGCGCGGAGGAGGAGGGAGGCC1131 nucleotide 
A
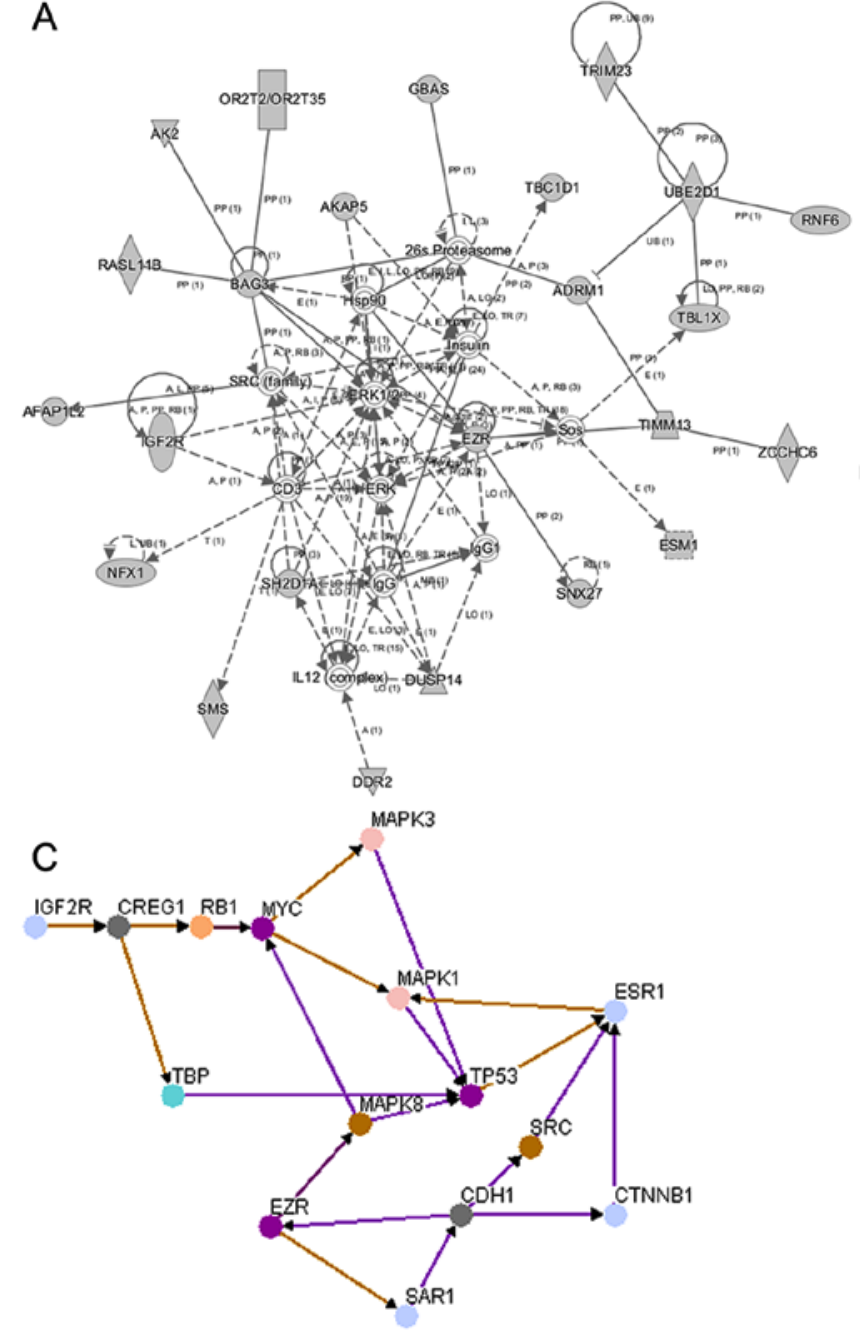

B

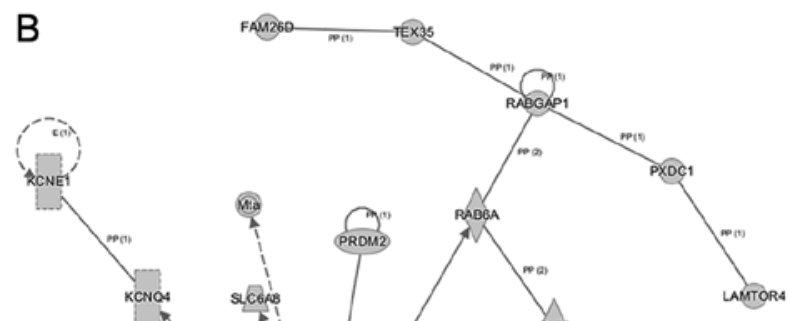

Figure 3. (A) INDEL network as analysed by IPA. (B) SNV network as analysed by IPA. (C) Protein-protein interaction network. Nodes represent hub proteins as analysed by IPA and their interacting proteins. INDEL, insertions-deletion; IPA, ingenuity pathway analysis; SNV, single-nucleotide variant.
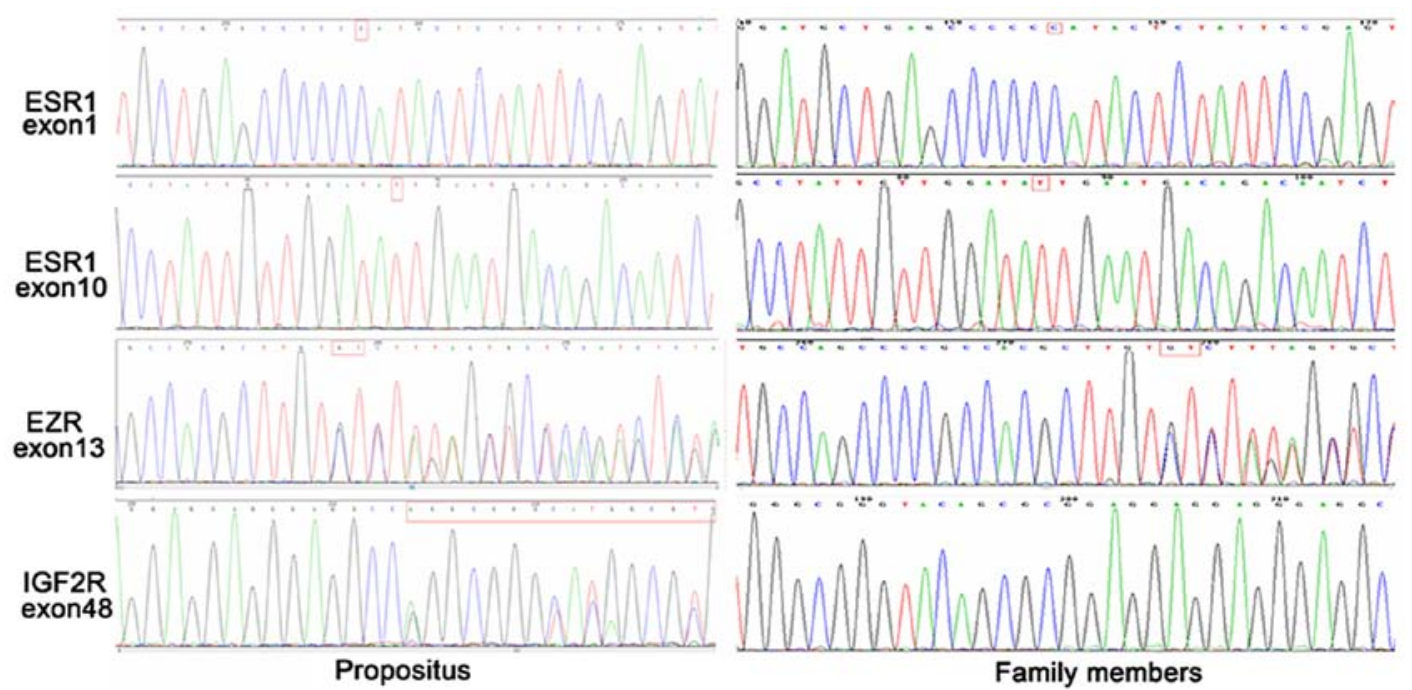

Figure 4. Sequencing diagram of the mutations in the ESR1, EZR and IGF2R genes of the propositus and his family members.

sequence in exon 48 of the IGF2R gene. ESR 1 and EZR carried the same mutations in other family members, while no consistent mutations were detected for IGF2R in other family members (Fig. 4). 

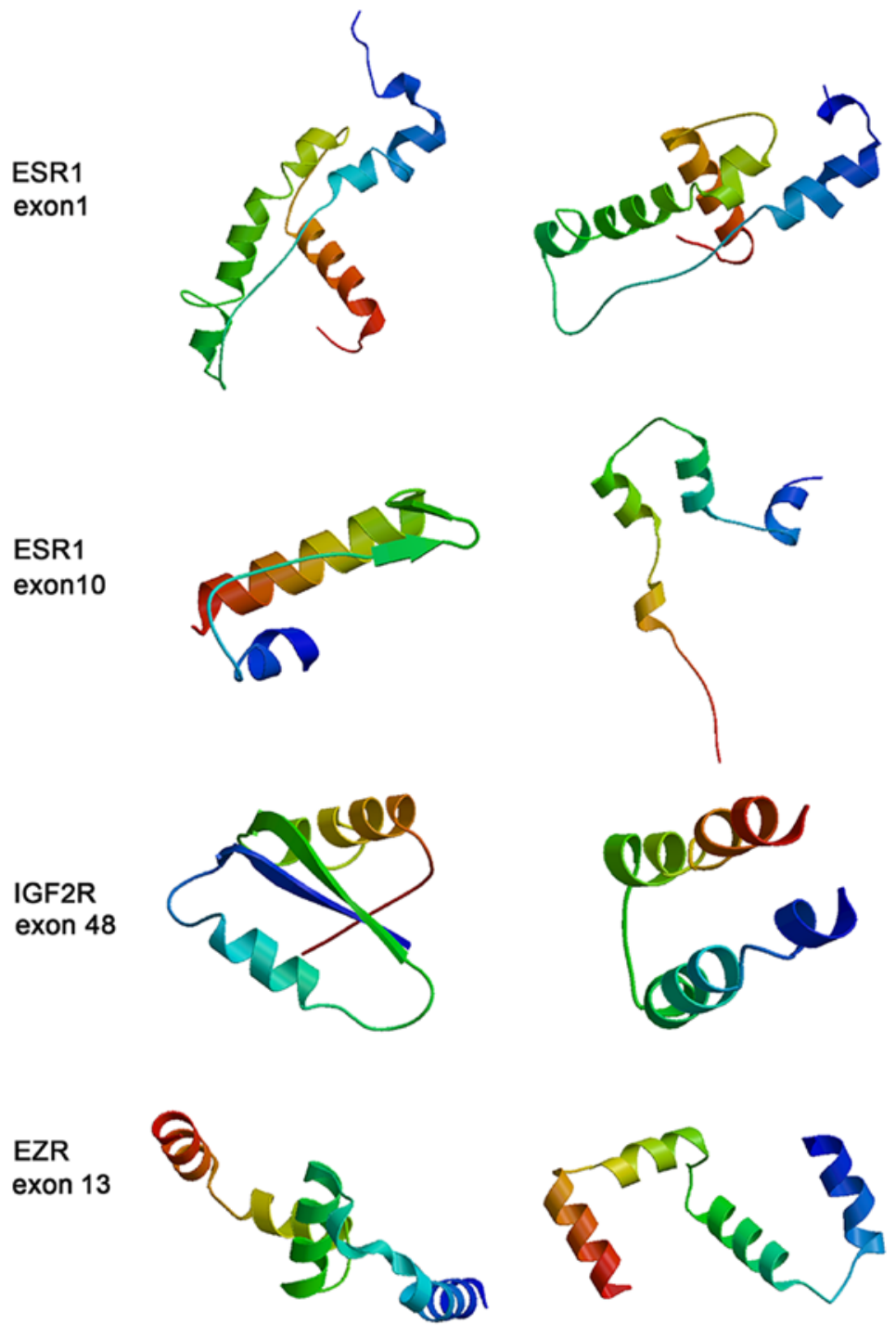

Normal

Mutant

Figure 5. Prediction of the protein structure before and after mutations by SWISS-MODEL.

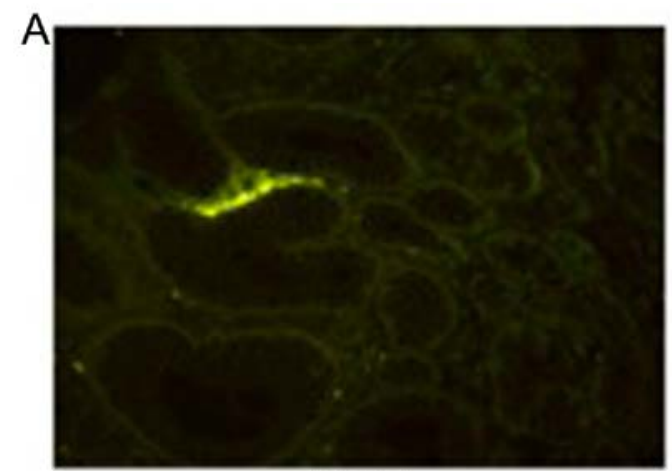

ESR1

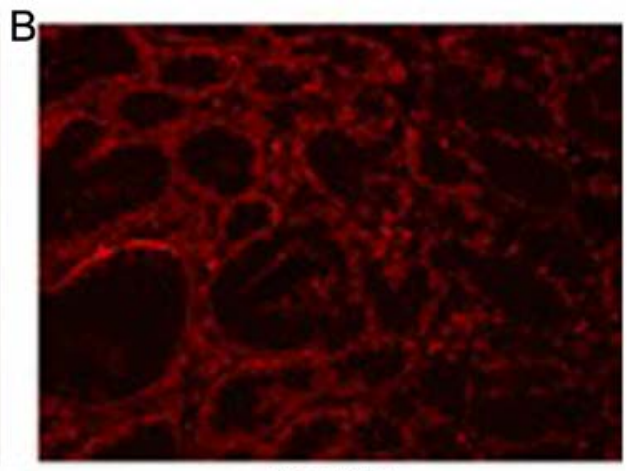

IGF2R

Figure 6. Immunofluorescence staining of (A) ESR1 and (B) IGF2R (magnification, x200).

Protein structure modelling. The protein structures before and after mutations were predicted by SWISS-MODEL. As illustrated in Fig. 5 the protein structures of ESR1, IGF2R and EZR became loose after mutation, particularly for ESR1 with a mutation in exon 10 .
Immunofluorescence staining of IGF2R and ESRI. The immunofluorescence staining of ESR1 and IGF2R was observed under the green and red channel, respectively. As displayed in Fig. 6 the green staining was less pronounced and not specific for ESR1. Bright red staining was observed throughout the tissue section for IGF2R. 


\section{Discussion}

According to Lauren's widely used histological classification, gastric cancer (GC) can be divided into intestinal and diffuse types of adenocarcinoma. Histopathologically, the Lauren GC intestinal histotype is more strongly associated with the GC familial history than the diffuse histotype (16). Incomplete intestinal metaplasia strongly increases the risk of GC and is regarded as a precursor of GC (17). Although the role of intestinal metaplasia in $\mathrm{GC}$ has been determined in previous studies (18) and several studies associate some genetic factors with GC among individuals with a family history of cancer (19), to date, the genetic cause for FIGC has not been well-identified. The identification of new markers predicting FIGC is important and has become the focus of intense research. Exome sequencing applied in the present study has contributed to shaping the complexity of cancers. The present study was designed to identify the putative predisposing gene defects underlying FIGC by comparing the mutation patterns with controls in a family with intestinal-type GC. In the present study, we reported an intestinal-type GC pedigree, displaying the features of family members harbouring GC with an intestinal histotype.

Based on the current dataset of exome sequencing, 2048 INDELs and 15819 SNVs were identified in the blood samples of subjects with a strong familial history of intestinal-type GC. Compared with the public exome sequencing data of the blood and tissue samples of GC patients, 74 and 2,089 overlapped genes harbouring INDELs and SNVs, respectively, were analysed. According to the GO functional enrichment analysis, the genes with INDELs were closely related to DNA packaging and nucleosome assembly, while SNV genes were enriched in cell-function-related BPs. Genes harbouring INDELs and SNVs may play different roles in the development and progression of FIGC. Pathway analysis revealed that genes harbouring SNVs were closely involved in cancer-related pathways, which proved that our findings were reliable and that genes harbouring SNVs may be centred to tumourigenesis from the intestinal metaplasia.

IPA network analysis revealed several hub genes of centrality. ERK1/2 had a remarkable centrality in the INDELs network, interacting with EZR and IGF2R. The ESR1-centred interaction network was constructed for SNVs. ERK1/2, belonging to the MAPK family, is expressed in mammalian cells (20). MAPKs, a family of mitogen-activated protein kinases, play regulatory roles in cell growth, differentiation and apoptosis (21). Accumulating evidence indicate that the activation of the MAPK/ERK signalling pathway is a common event in tumour development and invasion $(22,23)$. The ESR1 gene, encoding oestrogen receptor $\alpha$, is a well-known proto-oncogene. The activation of ESR1 induced ERK phosphorylation in a mouse spermatocyte-derived cell line, leading to apoptosis (24). In addition, IGF2R is a multiple ligand-binding cell surface receptor, the sequence of which corresponds to the bovine calcium-independent M6-P receptor. Insulin-like growth factor 2 regulates cell proliferation, apoptosis, migration and invasive ability and functions as a tumour suppressor. It has been reported that IGF2R also plays a key role in activating the downstream ERK/MAPK pathway (25). Ezrin-mediated early metastasis was reported in osteosarcoma and was partially dependent on the activation of the ERK/MAPK pathway (26). In the present study, the predicted protein-protein interaction network indicated that EZR played a regulatory role in the MAPK signalling pathway. Furthermore, previous evidence indicated that aberrant regulation of the MAPK pathway was closely associated with the development of cancer (27). Thus, we speculated that genes harbouring INDELs and SNVs perturbed the MAPK/ERK signalling pathway mediated by IGF2R and EZR, which further affected the downstream genes involved in cell apoptosis, differentiation and proliferation underlying FIGC development.

In the present study, the mutations of ESR1, EZR and IGF2R were identified in a family with intestinal-type GC by sequencing analysis. Most ESR1 mutations such as p.Leu536Gln, p.Tyr537Ser, p.Asp538Gly and D538G mutations (28-30) were identified in the ligand-binding domain (LBD) of oestrogen receptors. These mutations appear to be driver mutations, leading to a constitutively active form of ER that becomes oestrogen-independent (29). ESR1 genetic variations promote the development and progression of various cancers by altering oestrogen metabolism and play an important role in hormone binding, DNA binding and the activation of transcription to stimulate the alteration of the expression of downstream genes (30). Hypermethylation of ESR 1 is associated with a loss of expression of oestrogen receptor- $\alpha$, which may play a critical role in the carcinogenesis, development and prognosis of GC (31). IGF2R opposes the growth-promoting effects of IGF-2 and acts as a tumour suppressor gene for several cancers (10). It has also been demonstrated to be mutated in multiple human cancers $(32,33)$. IGF2R was identified to be mutated in exon 27, 28 and 40 for hepatocellular carcinomas $(34,35)$ and in exon 31 and 48 for breast cancer (36). When IGF2R is mutated, it loses its anti-oncogenic activity and neoplastic transformation may be caused by IGF 2 overaccumulation (33). IGF2R also inhibits the IGFR signalling pathway. The IGFR signalling pathway plays an important role in regulating cell proliferation, differentiation, apoptosis and development (37). As a multifunctional protein receptor, IGF2R can bind IGF2 at the cell surface and regulates the IGFR signalling pathway (10). Ezrin has been reported to have a crucial role in the dissemination of several tumours (38). However, EZR mutations in cancers have been rarely reported, particularly for GC.

To our knowledge, in the present study we reported for the first time a novel EZR deletion mutation in exon 13, ESR1 gene homozygous mutations in exon $1(216 \mathrm{G}>\mathrm{C})$ and exon 10 $(2234 \mathrm{C}>\mathrm{T})$ and an IGF2R insertion mutation in exon 48 of the intestinal-type GC family.

Protein models built by SWISS-MODEL revealed that the protein structure for ESR1, IGF2R and EZR had significant changes upon mutations. The genetic mutations may cause alterations in protein structure and affect the protein function, further altering the phenotype. Based on our findings, we speculated that the protein structure changes for ESR1, IGF2R and EZR may affect signal transduction upstream and downstream of the MAPK/ERK signalling pathway, which is related to tumourigenesis. We also speculated that the mutation of ESR1 affected protein structure and expression, leading to 
the dysregulation of oestrogen signalling pathways, which may contribute to tumourigenesis. Further research is warranted to prove this hypothesis.

Currently, it is recognized that patients with a familial history of GC and precancerous conditions and lesions of the stomach may benefit from periodic surveillance (39). Therefore, for individuals with genetic mutations, we recommend intensive endoscopic surveillance annually to ensure that there is no evidence of clinically significant lesions.

Several limitations of the present study should be mentioned. Firstly, the small sample size and number of specimens limited the validation of our results. Secondly, the protein functions affected by gene mutations were not deeply investigated. Our exploratory study seeking an association between genetic variation and subjects with a family history of GC indicated that ESR1, EZR and IGF2R are candidate mutations associated with FIGC. Further biological and clinical studies should be performed to ascertain the intricate mechanisms and clinical physiological relevance of correlation in terms of mutation biology, gastric tumourigenesis and therapeutic response.

In conclusion, exome sequencing of the members of a FIGC family outlined the pathogenesis and revealed that the driving mutations in ESR1, EZR and IGF2R play the hub roles in GC pathogenesis. These mutations show potential as candidate biomarkers and as therapeutic targets in the treatment of FIGC. The variants presented here have not been previously reported. The present study provided a novel insight into the pathogenesis of GC as well as guides for the counselling of predisposed individuals.

\section{Acknowledgements}

Not applicable.

\section{Funding}

The present study was supported in part by a grant from the National Natural Science Foundation of China (Key Program 81502401).

\section{Availability of data and materials}

The datasets used during the present study are available from the corresponding author upon reasonable request.

\section{Authors' contributions}

HC and JW conceived and designed the study. YZ and HW performed the experiments. JW wrote the paper. HC and JW reviewed and edited the manuscript. All authors read and approved the manuscript and agree to be accountable for all aspects of the research in ensuring that the accuracy or integrity of any part of the work are appropriately investigated and resolved.

\section{Ethics approval and consent to participate}

All procedures performed in studies involving human participants were in accordance with the ethical standards of the
Research Ethics Committee of The Third Affiliated Hospital of Nanjing University of Chinese Medicine and with the 1964 Helsinki declaration and its later amendments or comparable ethical standards. Informed consent was obtained from all patients or their relatives for the use of their tissues in the experimental procedures.

\section{Patient consent for publication}

Not applicable.

\section{Competing interests}

The authors declare that they have no competing interests.

\section{References}

1. Rugge M, Fassan M and Graham DY: Epidemiology of Gastric Cancer. Springer International Publishing, pp23-34, 2015.

2. Yaghoobi M, Bijarchi R and Narod SA: Family history and the risk of gastric cancer. Br J Cancer 102: 237-242, 2010.

3. Vogelaar IP, van der Post RS, Bisseling TM, van Krieken JHJ, Ligtenberg MJ and Hoogerbrugge N: Familial gastric cancer: Detection of a hereditary cause helps to understand its etiology. Hered Cancer Clin Pract 10: 18, 2012.

4. Caldas C, Carneiro F, Lynch HT, Yokota J, Wiesner GL, Powell SM, Lewis FR, Huntsman DG, Pharoah PD, Jankowski JA, et al: Familial gastric cancer: Overview and guidelines for management. J Med Genet 36: 873-880, 1999.

5. Carneiro F: Hereditary gastric cancer. Pathologe 33 (Suppl 2): S231-S234, 2012.

6. Oliveira C, Pinheiro H, Figueiredo J, Seruca R and Carneiro F: Familial gastric cancer: Genetic susceptibility, pathology, and implications for management. Lancet Oncol 16: e60-e70, 2015.

7. Nagarajan N, Bertrand D, Hillmer AM, Zang ZJ, Yao F Jacques PÉ, Teo AS, Cutcutache I, Zhang Z, Lee WH, et al: Whole-genome reconstruction and mutational signatures in gastric cancer. Genome Biol 13: R115, 2012.

8. Suleiman SH, Koko ME, Nasir WH, Elfateh O, Elgizouli UK, Abdallah MO, Alfarouk KO, Hussain A, Faisal S, Ibrahim FM, et al: Exome sequencing of a colorectal cancer family reveals shared mutation pattern and predisposition circuitry along tumor pathways. Front Genet 6: 288, 2015.

9. Sommer S and Fuqua SA: Estrogen receptor and breast cancer. Semin Cancer Biol 11: 339-352, 2001.

10. Schaffer BS, Lin MF, Byrd JC, Park JH and MacDonald RG: Opposing roles for the insulin-like growth factor (IGF)-II and mannose 6-phosphate (Man-6-P) binding activities of the IGF-II/Man-6-P receptor in the growth of prostate cancer cells. Endocrinology 144: 955-966, 2003.

11. Vaheri A, Carpén O, Heiska L, Helander TS, Jääskeläinen J, Majander-Nordenswan P, Sainio M, Timonen T and Turunen O: The ezrin protein family: Membrane-cytoskeleton interactions and disease associations. Curr Opin Cell Biol 9: 659-666, 1997.

12. Krämer A, Green J, Pollard J Jr and Tugendreich S: Causal analysis approaches in Ingenuity Pathway Analysis. Bioinformatics 30: 523-530, 2014.

13. Breitkreutz BJ, Stark C and Tyers M: Osprey: A network visualization system. Genome Biol 4: R22, 2003.

14. Gasteiger E, Gattiker A, Hoogland C, Ivanyi I, Appel RD and Bairoch A: ExPASy: The proteomics server for in-depth protein knowledge and analysis. Nucleic Acids Res 31: 3784-3788, 2003.

15. Pirooznia M, Nagarajan V and Deng Y: GeneVenn - A web application for comparing gene lists using Venn diagrams. Bioinformation 1: 420-422, 2007.

16. Bernini M, Barbi S, Roviello F, Scarpa A, Moore P, Pedrazzani C, Beghelli S, Marrelli D and de Manzoni G: Family history of gastric cancer: A correlation between epidemiologic findings and clinical data. Gastric Cancer 9: 9-13, 2006.

17. Filipe MI, Potet F, Bogomoletz WV, Dawson PA, Fabiani B, Chauveinc P, Fenzy A, Gazzard B, Goldfain D and Zeegen R: Incomplete sulphomucin-secreting intestinal metaplasia for gastric cancer. Preliminary data from a prospective study from three centres. Gut 26: 1319-1326, 1985. 
18. Cassaro M, Rugge M, Gutierrez O, Leandro G, Graham DY and Genta RM: Topographic patterns of intestinal metaplasia and gastric cancer. Am J Gastroenterol 95: 1431-1438, 2000.

19. Ghosh A, Hartge P, Purdue MP, Chanock SJ, Amundadottir L, Wang Z, Wentzensen N, Chatterjee $\mathrm{N}$ and Wacholder S: Assessing disease risk in genome-wide association studies using family history. Epidemiology 23: 616-622, 2012.

20. Cavalli V, Vilbois F, Corti M, Marcote MJ, Tamura K, Karin M, Arkinstall S and Gruenberg J: The stress-induced MAP kinase p38 regulates endocytic trafficking via the GDI:Rab5 complex. Mol Cell 7: 421-432, 2001.

21. Junttila MR, Li SP and Westermarck J: Phosphatase-mediated crosstalk between MAPK signaling pathways in the regulation of cell survival. FASEB J 22: 954-965, 2008

22. Reddy KB, Nabha SM and Atanaskova N: Role of MAP kinase in tumor progression and invasion. Cancer Metastasis Rev 22: 395-403, 2003

23. Dhillon AS, Hagan S, Rath O and Kolch W: MAP kinase signalling pathways in cancer. Oncogene 26: 3279-3290, 2007.

24. Chimento A, Sirianni R, Casaburi I, Ruggiero C, Maggiolini M, Andò $\mathrm{S}$ and Pezzi V: $17 \beta$-Estradiol activates GPER- and ESR1-dependent pathways inducing apoptosis in GC-2 cells, a mouse spermatocyte-derived cell line. Mol Cell Endocrinol 355 49-59, 2012.

25. Buchanan CM, Phillips AR and Cooper GJ: A novel two-chain IGF-II-derived peptide from purified $\beta$-cell granules. Growth Horm IGF Res 20: 360-366, 2010.

26. Khanna C, Wan X, Bose S, Cassaday R, Olomu O, Mendoza A, Yeung C, Gorlick R, Hewitt SM and Helman LJ: The membrane-cytoskeleton linker ezrin is necessary for osteosarcoma metastasis. Nat Med 10: 182-186, 2004.

27. Roberts PJ and Der CJ: Targeting the Raf-MEK-ERK mitogen-activated protein kinase cascade for the treatment of cancer. Oncogene 26: 3291-3310, 2007.

28. Robinson DR, Wu YM, Vats P, Su F, Lonigro RJ, Cao X, Kalyana - Sundaram S, Wang R, Ning Y, Hodges L, et al: Activating ESR1 mutations in hormone-resistant metastatic breast cancer. Nat Genet 45: 1446-1451, 2013.

29. Toy W, Shen Y, Won H, Green B, Sakr RA, Will M, Li Z, Gala K, Fanning S, King TA, et al: ESR1 ligand-binding domain mutations in hormone-resistant breast cancer. Nat Genet 45 : 1439-1445, 2013.
30. Schiavon G, Hrebien S, Garcia-Murillas I, Cutts RJ, Pearson A Tarazona N, Fenwick K, Kozarewa I, Lopez-Knowles E, Ribas R, et al: Analysis of ESRI mutation in circulating tumor DNA demonstrates evolution during therapy for metastatic breast cancer. Sci Transl Med 7: 313ra182, 2015.

31. Woo IS, Park MJ, Choi SW, Kim SJ, Lee MA, Kang JH, Hong YS and Lee KS: Loss of estrogen receptor-alpha expression is associated with hypermethylation near its ATG start codon in gastric cancer cell lines. Oncol Rep 11: 617-622, 2004.

32. Kong FM, Anscher MS, Washington MK, Killian JK and Jirtle RL: $M 6 P / I G F 2 R$ is mutated in squamous cell carcinoma of the lung. Oncogene 19: 1572-1578, 2000.

33. Oka Y, Waterland RA, Killian JK, Nolan CM, Jang HS, Tohara K, Sakaguchi S, Yao T, Iwashita A, Yata Y, et al: M6P/IGF2R tumor suppressor gene mutated in hepatocellular carcinomas in Japan. Hepatology 35: 1153-1163, 2002.

34. Yamada T, De Souza AT, Finkelstein S and Jirtle RL: Loss of the gene encoding mannose 6-phosphate/insulin-like growth factor II receptor is an early event in liver carcinogenesis. Proc Natl Acad Sci USA 94: 10351-10355, 1997.

35. De Souza AT, Hankins GR, Washington MK, Orton TC and Jirtle RL: M6P/IGF2R gene is mutated in human hepatocellular carcinomas with loss of heterozygosity. Nat Genet 11: 447-449, 1995.

36. Hankins GR, De Souza AT, Bentley RC, Patel MR, Marks JR, Iglehart JD and Jirtle RL: M6P/IGF2 receptor: A candidate breast tumor suppressor gene. Oncogene 12: 2003-2009, 1996.

37. $\mathrm{Yu} \mathrm{H}$ and Rohan T: Role of the insulin-like growth factor family in cancer development and progression. J Natl Cancer Inst 92: 1472-1489, 2000.

38. Mäkitie T, Carpén O, Vaheri A and Kivelä T: Ezrin as a prognostic indicator and its relationship to tumor characteristics in uveal malignant melanoma. Invest Ophthalmol Vis Sci 42: 2442-2449, 2001

39. Hirota WK, Zuckerman MJ, Adler DG, Davila RE, Egan J, Leighton JA, Qureshi WA, Rajan E, Fanelli R, Wheeler-Harbaugh J, et al; Standards of Practice Committee, American Society for Gastrointestinal Endoscopy: ASGE guideline: The role of endoscopy in the surveillance of premalignant conditions of the upper GI tract. Gastrointest Endosc 63: 570-580, 2006. 